\title{
The Improved Genetic Algorithm for Distribution Network Life Cycle Costs Planning
}

\author{
Pei Hao ${ }^{1, a}$, Jingang Shi ${ }^{2, b}$, Chenrui $\mathrm{Li}^{2, \mathrm{c}}$ \\ ${ }^{1}$ Shijiazhuang Science \& Technology Cooperation and Innovation Platform Center, Shijiazhuang \\ 050026, China; ${ }^{2}$ Xining Power Supply Company 810000 China \\ ahopehpy@sina.com, 'bhijingang03@163.com, chenrui7227@126.com
}

Keywords: Life cycle costs; distribution network planning; clustering crowding niche genetic algorithm; radial distribution network.

\begin{abstract}
The life cycle economy of the power distribution network planning scheme is becoming more and more important in power markets. The model of the distribution network planning and the configuration of switches based on the life cycle cost (LCC) of equipment was presented. The main objective is to minimize the total life cycle costs which including the initial investment, operation and maintenance cost, outage cost and disposal cost of planning scheme. The clustering crowding niche genetic algorithm is used to get the optimum planning scheme. Finally, the feasibility and effectiveness of the method are verified by the result of applying it to a test system.
\end{abstract}

\section{Introduction}

The distribution network planning is essentially a discrete, nonlinear, multi-stage and multi-objective combinatorial optimization problem, and its aim is to meet user demand under the premise of looking for a set of optimal decision variables (type of feeder kinds and paths and the switch position, etc.), minimizing the investment planning, network loss costs and the user power outage loss. For a long time, scholars have done a lot of research on this issue. The distribution feeder lines optimization based on minimum costs was proposed in paper [1]. The artificial intelligence algorithms were introduced to the distribution network planning in papers[2-5]. The methods mentioned above can not reflect the economy of planning scheme's life cycle costs. The paper [1] considered the costs at all stages of the feeder path optimization problem, but did not consider optimal configuration of the switch in distribution network planning.

In this paper, a new distribution network planning optimization method that based on the minimum life cycle costs (LCC) are proposed. The traditional distribution network planning with minimal system investment is to meet the target reliability in the level of power load, and ignore the operation, maintenance, refurbishment and retirement costs, even lack the overall consideration for planning scheme within the social LCC (Outage costs, environmental costs). For the long-term point of view, planning scheme's operation costs, maintenance costs and social costs are much more than its construction costs. The level of construction costs will have great influence on the future operation costs, maintenance costs and social costs, and high construction costs may reduce the future operation maintenance costs significantly, so as to make the planning scheme's costs reduce throughout the life cycle. Therefore it is necessary to do distribution network planning based on the life cycle perspective[6].

\section{The distribution network LCC modem}

The objective of the distribution network LCC planning is to optimize the economical benefits in life cycle, seek the optimal project's LCC on the premise of safety, effectiveness and environmental efficiency, determine the best distribution network planning scheme, and create the maximum economic benefit, social and environmental benefits. The core is to analysis and calculates the LCC of planning scheme, and makes decision according to the LCC. The LCC are the system total costs from design to decommissioning, and mainly include: one-time investment costs $C_{\mathrm{I}}$; operation costs 
$C_{\mathrm{O}}$; maintenance costs $C_{\mathrm{M}}$; failure costs $C_{\mathrm{F}}$; disposal costs $C_{\mathrm{D}}$. Details calculation model are as follows.

\subsection{One-time investment costs $C_{I}$}

The $C_{I}$ mean that the costs paid in the duration of planning design and construction of the distribution network. It mainly includes the design costs, the equipment investment costs, and construction and installation costs. Design costs mainly includes: research costs, feasibility study costs, preliminary design costs, construction design costs, and so on; Equipment investment costs mainly includes equipment purchase costs, construction costs, installation fees, construction loan interest and other costs.

$$
C_{I}=C_{\mathrm{ec}}+C_{\mathrm{dc}}=\sum_{i \in M} C_{\mathrm{ed}}+C_{\mathrm{dc}}
$$

Where, $C_{\mathrm{ec}}$ is all the equipment investment costs; $C_{\mathrm{ec} i}$ is the $i$ th equipment's purchase costs and installation costs ; $M$ is the quantity of equipments; $C_{\mathrm{dc}}$ is the design costs of the distribution network.

\subsection{Operation costs $C_{0}$}

The $C_{\mathrm{O}}$ are the sum of all costs required during the operation period. It includes equipment loss costs, operation staff training costs, and so on. Equipment loss mainly includes line loss and transformer loss.

1) electric energy loss costs $C_{\text {eo }}$

The power loss is calculated by the method of maximum hours of the load loss. Under the condition of knowing the load distribution, the power loss $\Delta P_{\max }$ can be calculated by the power flow calculating, and the maximum load loss of time $\tau_{\max }$ is easy to be looked up according to the maximum load utilization hours $T_{\max }$ and the power factor. The computational formula as follows:

$$
C_{\mathrm{eo}}=\Delta W_{\mathrm{S}} \times C_{\mathrm{P}}=\Delta P_{\max } \times \tau_{\max } \times C_{\mathrm{P}}
$$

Where, $\Delta W_{s}$ annual energy loss; $C_{\mathrm{p}}$ is the price of power.

2) Operators training costs $C_{\mathrm{tc}}$. Taking a certain percentage of the initial investment.

\subsection{Maintenance costs $C_{M}$}

The $C_{M}$ include maintenance staff costs, equipment maintenance and other expenses every year, throughout the entire life cycle. As the power equipment maintenance system, the equipment maintenance cycle and maintenance costs are relatively stable. The $C_{\mathrm{M}}$ can be determined as follow.

$$
C_{M}=\sum_{i \in M} \lambda_{i} \times R C_{i}
$$

Where $\lambda_{i}$ is the average maintenance times related to the ith equipment, $R C_{i}$ is the average maintenance costs unit time to the $i$ th equipment which include equipment maintenance costs and maintenance staff costs.

\subsection{Failure costs $C_{F}$}

Failure costs are the economic loss of the electric network and users caused by failure outage. They mainly include outage costs, social influence costs, etc.

1) Outage costs $C_{\mathrm{oc}}$

Outage costs are losses when electricity supply is not completely reliable or anticipated reliable. The $C_{\mathrm{oc}}$ are calculated by the ratio of output value to unit electric energy consumption proposed in reference [7]. Outage costs during the study period could be calculated as follows:

$$
C_{\mathrm{oc}}=\sum_{i=1}^{m} R_{\mathrm{oven}} \times \mathrm{EENS}_{i}
$$

Where $m$ is the number of the load node in the distribution network, $R_{\text {oven }}$ is the ratio of output value to unit electric energy consumption, unit is yuan/kwh, EENS ${ }_{i}$ is the $i$ th node expected energy not supplied during the study period(kwh/period).

2) social influence costs $C_{\mathrm{OS}}$

The $C_{\mathrm{OS}}$ mainly include environmental pollution control costs, social coordination costs, etc.

\subsection{Disposal costs $C_{\mathrm{D}}$}

The $C_{\mathrm{D}}$ refer to the costs required in the disposal process. It not only include the necessary remedial treatment (such as waste disposal, etc.) costs, but also equipment residual value. 


$$
C_{D}=\sum_{i \in M}\left(C_{i e d}-C_{i e r}\right)
$$

Where, $C_{i e d}$ is the costs to deal with the $i$ th equipment; $C_{i e r}$ is the residual value of the $i$ th equipment .

\section{Distribution network planning based on life cycle costs}

The planning example does not involve the substation planning problems, and the proposed method based on the existing network structures and parameters is used to select lines reasonably and optimize the distribution circuit breaker equipment configuration.

\subsection{Mathematical model of distribution network planning}

The objective function is to obtain the minimum life cycle costs of distribution network planning scheme. The optimization model for distribution network planning is established with the constraints of reliability and power flow of the system as follows:

$$
\min L C C=\left\{\begin{array}{lr}
C_{I}+\left(\mathrm{C}_{\mathrm{O}}+C_{M}+C_{F}\right) \times \mathrm{PV}_{\text {sun }}+C_{D} \times P V+\mathrm{U}_{1} \mathrm{~L} & \text { radial network } \\
U_{2} & \text { unradial network }
\end{array}\right.
$$

Subject to the following constraints:

1) $A P=D$

2) $P_{i}<P_{\text {imax }}$

3) The operation mode of Radial network

Where $A$ is the node incidence matrix; $P$ is the network power flow; $D$ is the load demand; $P_{i}$ is the branch power flow; $P_{\text {imax }}$ is the maximum allowable capacity of the branch; LCC is the life cycle costs for the equipment; $P V=1 /(1+r)^{N}$ is the discount factor; $P V_{\text {sum }}=\left((1+r)^{N}-1\right) /\left(r(1+r)^{N}\right)$ is the sum of present value of the annual operation costs; $N$ is the project life cycle; $r$ is the discount rate; $U_{1}$ is the overload penalty coefficient; $L$ is the overload of power network (that is more than the maximum allowable load of feeders) and its value can be obtained by the calculation of the network of power flow; $U_{2}$ is the penalty value of unradial network which should be set max enough to eliminate the unfeasible solution.

The flow constraints can be achieved by the power flow calculation based on forward and backward substitution methods [8].

\subsection{Solution of mathematical model}

Distribution network planning optimization is a multi-objective, multi-stage, discrete, nonlinear and constrained mixed integer programming problem.

Excluding niche and improved genetic algorithm has been widely used in power system optimization problems [9]. The main idea is: First the hamming distance between every two individuals in the group (or Euclidean distance, etc.) is compared. If the distance is less than pre-specified distance L, then the fitness of both is compared and a strong penalty function is imposed to the smaller fitness individual, which greatly reduces its fitness. Thus, for the distance of two individuals within the L, the smaller fitness individual after treatment becomes worse, and with greater probability of being eliminated later in the evolutionary process. In other words, it realizes a niche genetic algorithm. To some extent, the existing niche technology can maintain the population diversity, and has some global optimization capability. But these niche technology optimization effect and computing speed greatly influenced by the niche number, in distribution network planning often leads to optimal results with larger volatility. The basic reason is the existing algorithm can't establish and maintain the stable of niche.

The purpose of clustering is to set a large number of data points divided into several categories, making each class to maximize the similarity between the data, different types of data with the maximum difference between the data. Commonly the class of clustering criteria includes minimizing the sum of squares of the distance within the class and maximizing the sum of squares of the distance between the classes. The sum of squares of the distance within the class and the criterion are suitable for the situation of the individual closes to each other in the clustering, and the sum of 
squares of the distance between the class and the criterion for the occasion with obvious differences between each cluster. In determining the clustering center, this paper adopts the criterion of the maximum sum of squares of the distance between the classes; in determining the classification of all individuals, using the criterion of the minimum sum of squares of the distance within the classes [10].

The cluster analysis combined with the exclusion mechanism [10], can effectively search for global optimal point, at the same time they can adjust the minimum clustering radius, control the number of converged niche, and avoid finding invalid extreme value point.

1) Coding scheme

The distribution network can be expressed as the combination of lines, by changing the state of the route to change the topological structure of the network. 0 means that the line is not selected, and 1 means that the line is selected, each line occupies a gene on chromosome, chromosome length equal to the total number of planned routes.

2) The initialization of swarms

Considering the radial distribution, we will get plenty of infeasible solution if we use the random method to initialize population, it will affect the optimal speed. Based on the graph theory knowledge [13], this paper generate optional network spanning tree by random, as the initial solution of the genetic optimization algorithm, it make the initial solution to be radial feasible solution.

3) Judgment subprogram of radial network

After genetic crossover and mutation, the scheme is not necessarily a radial network, therefore, it need to judge the network, and layered breadth-first search algorithm is used in this paper. At fist we choose the substation for the root node, search all the child nodes connected to the root node as the root node for the next search, and then search the child nodes of the root nodes, and so on, until all the nodes traversed. If a node's line number is found large than 1 in the process, it is judged to be a non-radial network, and a rather large penalty value should be given to the fitness value.

4) Algorithm basic steps

a) Randomly generate the initial individual and make the initial population, and set the cluster distance; b) Order the fitness of all individual values in the population by descending order, set the highest fitness individual as the cluster center, and classify some individuals to the center according to the distance of individuals within the class. c) Repeat steps in the remaining individuals 2) until the end of classification. d) Carry on the fitness sharing of niche genetic algorithm in the territory of niche, the concrete process sees the paper [4]. e) Determine the termination condition, not satisfied to return the step (2), if meet the terminating condition, output the result, and the algorithm ends.

\section{Application}

This paper takes a feeder of IEEE-RBTS bus- 2 for testing. The testing network is shown in figure 1 . In the figure dotted line indicates a potential new route, the total number is 28 . Lp1 Lp7 are the load node, other nodes are branch nodes. The main parameters are provided in [1, 14]. Population size is set to 200 , the maximum number of iterations is 50 , Electricity price is $c_{p}=0.6$ yuan $/ \mathrm{kWh}$, discount rate is $\mathrm{r}=6 \%$, ratio of output value to unit electric energy consumption is $9.65 \mathrm{yuan} / \mathrm{kWh}$, transformer reference price is 16.08 ten thousand yuan, isolating switch reference price is 0.223 ten thousand yuan, fuse reference price is 0.045 ten thousand yuan, main feeder reference price is 20 ten thousand yuan ,branch line reference price is 0.045 ten thousand yuan, life of planning scheme is 20years.

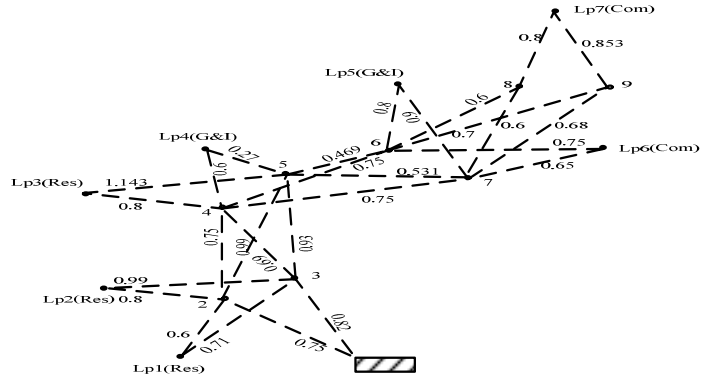

Figure 1. Test distribution network 
In this paper, the optimal program was development with Visual studio2005. Calculate 6 different connection modes, these connection modes are: 1) primary feeder with isolating switch, with branch line protection, alternative supply and repair of transformer; 2) primary feeder without isolating switch, without branch line protection, no alternative supply and repair of transformer; 3) primary feeder without isolating switch, with branch line protection, no alternative supply and repair of transformer; 4) primary feeder with isolating switch, without branch line protection, alternative supply and repair of transformer; 5) primary feeder with isolating switch, with branch line protection, alternative supply and replacement transformer; 6) primary feeder with isolating switch, without branch line protection, no alternative supply and repair of transformer. The result of optimal path for distribution network planning scheme is show in table 1.The result of LCC calculation for 6 different connection modes corresponding to planning scheme are shown in table 2.

Tab.1 The results of the optimal path planning

\begin{tabular}{ccll}
\hline Scheme & New lines \\
\hline 5 & 1-2, 2-Lp1, 2-LP2, 2-4, 4-Lp3, 4-Lp4, 4-7, 7-Lp5, 7-Lp6, 7-8, 8-Lp7 \\
$1,2,3,4,6$ & 1-2, 2-Lp1, 2-LP2, 2-4, 4-Lp3, 4-Lp4, 4-6, 6-Lp5, 6-Lp6, 6-8, 8-Lp7 \\
\hline
\end{tabular}

Tab.2 The LCC of the distribution network planning scheme

\begin{tabular}{ccccccc}
\hline costs $(10 \mathrm{k} Y)$ & 1 & 2 & 3 & 4 & 5 & 6 \\
\hline$C_{\mathrm{I}}$ & 232.57 & 231.36 & 231.68 & 232.25 & 343.92 & 232.03 \\
$C_{\mathrm{O}}$ & 266.35 & 266.35 & 266.35 & 266.35 & 266.35 & 266.35 \\
$C_{\mathrm{M}}$ & 80.16 & 79.75 & 79.86 & 80.06 & 118.55 & 79.98 \\
$C_{\mathrm{F}}$ & 157.99 & 953.42 & 169.30 & 311.42 & 42.818 & 620.26 \\
$C_{\mathrm{D}}$ & 0.7256 & 0.7218 & 0.7228 & 0.7246 & 1.0730 & 0.7239 \\
$L C C$ & 737.80 & 1531.6 & 747.91 & 890.80 & 772.71 & 1199.3 \\
\hline
\end{tabular}

From table 1 , the optimal route planning scheme is selected by the minimum life cycle cost, for different route schemes, the equipment and reliability of distribution network are different, therefore the investment costs, operation maintenance costs, failure costs and environmental costs are different. So, the life cycle costs are different. So, different mode of connection have different optimal planning path. For example, the optimal route planning scheme 5 is different of other schemes.

If only considers the initial investment capital of power grid project in the planning scheme optimization, we may choose a small initial capital investment of supply scheme. But from the perspective of long-term, the sum of operating costs, maintenance costs, failure costs of planning scheme are much higher than its construction costs, so it should generally consider the life cycle costs in distribution network planning program. Regarding scheme 5, because of its high system reliability, the reduction of failure costs is less than the increase investment and operation costs, by increasing stand-by transformer to improve the reliability, the LCC is not the optimization. Although planning scheme 5 has the highest reliability, it is not the best economic and reliable scheme. The scheme 1 is the optimal one based on minimum life cycle costs of power network, which compromises the network's security and economy by the optimal path and switch configuration.

\section{Conclusion}

This paper has applied the life cycle costs theory to the optimization of the distribution network planning. According to the operational characteristics of distribution network, the detailed life cycle costs calculation model of distribution network planning is established. The objective function based on the optimal life cycle costs is established, and the clustering crowding niche genetic algorithm is used to optimize the distribution network planning schemes. According to the analysis to the example, this paper gives the optimal planning and distribution network switch arrangement. The method is approved to be feasible and effective, and has further improved the application of the life-cycle costs management in power system planning project. 


\section{References}

[1]Yang Wei, Wang Lina. Distribution network structure planning based on improved immune genetic algorithm, joumal of chongqing university. Vol 30 no 28-31,2007.

[2] Zhang Liying, Fan Mingtian, A new model and methodology for distribution network integration planning, Proceedings of the CSEE, vol 24, No. 6, pp. 59-64, 2004.

[3] Kong Tao, Cheng Haozhong, Xu Tongyu, Urban Medium-Voltage Distribution Network Planning Based on ComGIS Network Analysis and Multi-objective Genetic Algorithm. Proceedings of the CSEE, vol 29, No. 19, pp. 49-55, 2008.

[4] Wang Tianhua, Wang Pingyang, Fan Mingtian, Optimal Multi-stage Distribution Planning Using Evolutionary Algorithm, Proceedings of the CSEE, vol 20, No. 3, pp. 34-38, 2000.

[5] Diaz-Dorado E, Cidras J, Miguez E, Application of evolutionary algorithms for the planning of urban distribution networks of medium voltage, IEEE Transactions on Power Systems, vol 17, No. 3 , pp. 879-884, 2002.

[6]Hua Chenbing, Huang Zhenhua, Zhang Gang,et al. Power distribution network optimal planning based on improved genetic algorithm,shandong dianli jishu. No. 42, pp. 16-18, 2015.

[7] Guo Yongji, Power System Reliabilituy Analysis, Tsinghua University Press, BeiJing, 2003.

[8] Yan Wei, Liu Fang, Wang Guanjie. et al, Layer-by-layer Back/forward Sweep Method For Radial Distribution Load Flow, Proceedings of the CSEE, vol 23, No. 8, pp. 76-80, 2003.

[9] Zhang Jingping, Liang Zhirui, SU Hai-feng, et al. Research on reactive power optimization of distribution network based on the improved crowding niche genetic algorithm[J].RELAY, 2007,35(10):19-22.

[10]YU Xinjie, WANG Zanji. A new clustering method and its applications on multimodal optimization[J]. J T singhua U niv (Sci \& Tech), 2001(45):159-161.

[11] Ma Xiufan, Cui Huanjun, An Improved Genetic Algorithm for Distribution Network Planning With Distributed Generation, Transactions of China Electrotechnical Society, vol.26, No.3,pp.176-180,2011.

[12] Zhang Yaxuan, Yan Cui-qun, Tang Wei,etal.The middle-voltage distribution network planning of new substation based on improved genetic algorithm,vol 39,No.4,pp.74-78,2011.

[13]Liu Lu, Wang Hejie, Cheng Haozhong, etal, Economic Evaluation of Power Systems Based on Life Cycle Cost, Automation of Electric Power Systems, Vol.36,No.15,pp.45-50,2012. 